\title{
Tesauros de Ciencias Experimentales y Tecnologías de la Información y la Comunicación en Internet
}

\section{Experimental Sciences and Information and Communication Technology Thesauri in Internet}

\author{
Gonzalo Mochón Bezares*, Ángela Sorli Rojo**
}

El presente trabajo completa una serie de artículos sobre tesauros de acceso libre presentes en Internet iniciada en esta misma revista en enero de 2007. En esta última entrega se recogen tesauros sobre ciencias experimentales, tecnologías de la información y las comunicaciones y disciplinas afines encontrados en la Red. Como en los anteriores artículos recopilatorios, los tesauros se muestran por orden alfabético de materias, situando a los de ciencias en general en primer lugar. Las URLs de los recursos comentados han sido consultadas a lo largo de los meses de octubre y noviembre de 2008.

\section{Generales}

\section{Tesauro Spines}

bttp://thes.cindoc.csic.es/index_SPIN_esp.html

La versión original del Tesauro SPINES (Sistema de Intercambio de Información sobre Políticas Científicas) fue elaborada entre los años 1972 y 1975 como herramienta de intercambio de información en el marco de un programa realizado por la UNESCO. Dicho plan tenía como fin facilitar el manejo e intercambio internacional de documentos y datos relativos a políticas científicas, investigación científica y transferencia de tecnología, tanto a nivel gubernamental como a nivel institucional. Aparte de esta finalidad primigenia, este tesauro ha sido utilizado

\footnotetext{
* Aspy System.

** IEDCYT.-CSIC.

Correo-e: asorli@cindoc.csic.es

Recibido: 19-12-2008.
} 
por numerosas instituciones que lo han destinado al tratamiento de la documentación sobre política científica y desarrollo económico y social.

La versión española consultada, que ha sido realizada por personal del Instituto de Ciencia y Tecnología (ICYT) sobre la versión en inglés publicada en 1984, contiene más de 10.000 términos entre descriptores y no descriptores. Su consulta puede realizarse visitando las áreas temáticas del índice jerárquico; navegando por el índice alfabético, en cual se distinguen tipográficamente los términos admitidos de los no admitidos; o utilizando el buscador (enlace búsquedas dentro del apartado consultas al tesauro).

Esta última herramienta permite la consulta de palabras o fragmentos de palabras, siendo el símbolo \% el carácter de sustitución o truncamiento empleado. La información que se ofrece sobre cada término incluye las relaciones semánticas del mismo marcadas como hiperenlaces, y su traducción al francés y al inglés. En la página web no se ofrece información sobre la fecha de la última actualización del tesauro.

\section{Arquitectura-Construccion-Ingenieria}

\section{Canadian Thesaurus of Construction Science and Technology bttp://irc.nrc-cnrc.gc.ca/thesaurus/toc-thesaurus.html}

La creación del Canadian Thesaurus of Construction Science and Technology (TC/CS) se debe al IF Research Group, dirigido por Colin H. Davidson, de la Universidad de Montreal, el cual actuó por encargo del Departamento de Industria y Comercio del Gobierno de Canadá. Sin embargo, la presente versión electrónica, realizada en 1995, ha corrido a cargo de Dana J. Vanier y Chenchao Chi, del National Research Council de Canadá. Este tesauro contiene más de 15.000 términos en inglés sobre construcción, tecnologías aplicadas a la construcción y ciencias afines. La consulta se puede hacer en un índice alfabético de términos, en el que no hay forma de distinguir los términos admitidos de los no admitidos. También se puede consultar utilizando la herramienta Google Custom Search, que sólo permite la recuperación por palabras completas. La información ofrecida sobre cada descriptor incluye sus relaciones semánticas indicadas de una forma algo peculiar, lo que puede despistar al usuario, y su traducción al francés. La última versión es de 1998.

\section{Tesauro de Ingeniería Hidráulica \\ bttp://bispagua.cedex.es/documentacion/tesauro/Tes bidro/tesauro. php?localizacion=Tesauro\%20Ingenier\%EDa\%20Civil}

El sitio web del Centro de Estudios y Experimentación de Obras Públicas (CEDEX) recoge el Tesauro de Ingeniería Hidráulica, desarrollado en el marco de un Convenio de colaboración entre el CEDEX y la Dirección General de Obras 
Hidráulicas del extinto Ministerio de Obras Públicas, Transportes y Medio Ambiente, con el fin de suplir la falta de vocabularios controlados sobre esta materia. La finalidad de este tesauro es garantizar la calidad de los procesos de indización y recuperación de información de distintas bases de datos. En la confección de este tesauro en lengua española colaboró personal del CEDX y del CINDOC (CSIC). El Tesauro de Ingeniería Hidráulica consta de 3.105 términos, de los cuales 2.932 son descriptores y 173 no descriptores. El contenido se puede consultar en un índice jerárquico que contiene todo el árbol jerárquico con los descriptores marcados como hiperenlaces, y en un índice conceptual en el que se recogen todos los términos por orden alfabético y la información de las relaciones semánticas de cada término marcadas como hiperenlaces.

\section{Astronomía}

\section{Astronomy Thesaurus Index}

\section{bttp://msowww.anu.edu.au/library/thesaurus/}

El sitio web de la Research School of Astronomy and Astrophysics de la Universidad Nacional de Australia dispone de un tesauro sobre astronomía, el Astronomy Thesaurus, que se puede consultar en cinco lenguas distintas: inglés, francés, alemán, italiano y español. El Astronomy Thesaurus, elaborado por Robyn M. Shobbrook y Robert R. Shobbrook, contiene 2.933 términos, de los cuales 2.536 son descriptores y 397, no descriptores. La consulta de este tesauro se puede realizar a través de un índice alfabético en el que se distingue la categoría de los términos por su grafía, o bien navegando por una lista jerárquica algo caótica que presenta un gran número de términos cabecera. Tanto los descriptores como los no descriptores se presentan marcados como hiperenlaces, lo cual facilita la consulta. El contenido del tesauro no se actualiza desde 1995.

\section{Defensa}

\section{Center for Army Lessons Learned Thesaurus bttp://usacac.army.mil/cac2/call/index.asp}

El Combined Arms Center (CAC) es un centro de instrucción militar para oficiales del ejército de los Estados Unidos. Dentro del apartado de recursos del sitio web del CALL podemos encontrar un vocabulario controlado destinado a un uso militar: el CALL Thesaurus. Se trata de un tesauro de gran magnitud que contiene 32.274 términos en inglés, principalmente sobre temática militar. Se incluye también como apéndice una lista con 862 acrónimos de temática militar. La consulta del CALL Thesaurus puede realizarse a través del buscador, que trunca automáticamente los términos consultados, o por medio de un índice alfabético en el que no hay diferencia gráfica entre los términos admitidos y los no 
admitidos. La información sobre cada término incluye los términos que conforman sus relaciones semánticas marcados como hiperenlaces. Se echa en falta un índice sistemático que permita al usuario hacerse una idea de las diferentes áreas tratadas en este macrotesauro.

\section{Public Technical Reports-Thesaurus bttp://www.dtic.mil/dtic/search/tr/str/thesaurus.html}

La entidad norteamericana Defense Technical Information Center (DTIC) es una empresa especializada en consultoría sobre defensa que actúa como mediadora entre los contratistas y las agencias gubernamentales de los Estados Unidos. Para facilitar la consulta por materias de esta colección, se presenta un vocabulario controlado sobre defensa: el DTIC Thesaurus. Este vocabulario controlado es un macrotesauro en lengua inglesa sobre defensa y otras materias que contiene 16.934 descriptores. La consulta de su contenido puede hacerse seleccionando la letra inicial de un término en el índice alfabético (operación bastante lenta, por cierto) o introduciendo términos en el buscador, que permite el uso del asterisco (*) como símbolo de truncamiento al principio y al final de los términos, así como el uso de los operadores booleanos and y or. La información que se muestra sobre cada descriptor incluye las relaciones semánticas del mismo e indicaciones de restricción de uso o de combinación con otros términos. Los descriptores se presentan siempre marcados como hiperenlaces, lo que en un tesauro de estas dimensiones es de agradecer. La última modificación del contenido de este macrotesauro se llevó a cabo en junio de 2008.

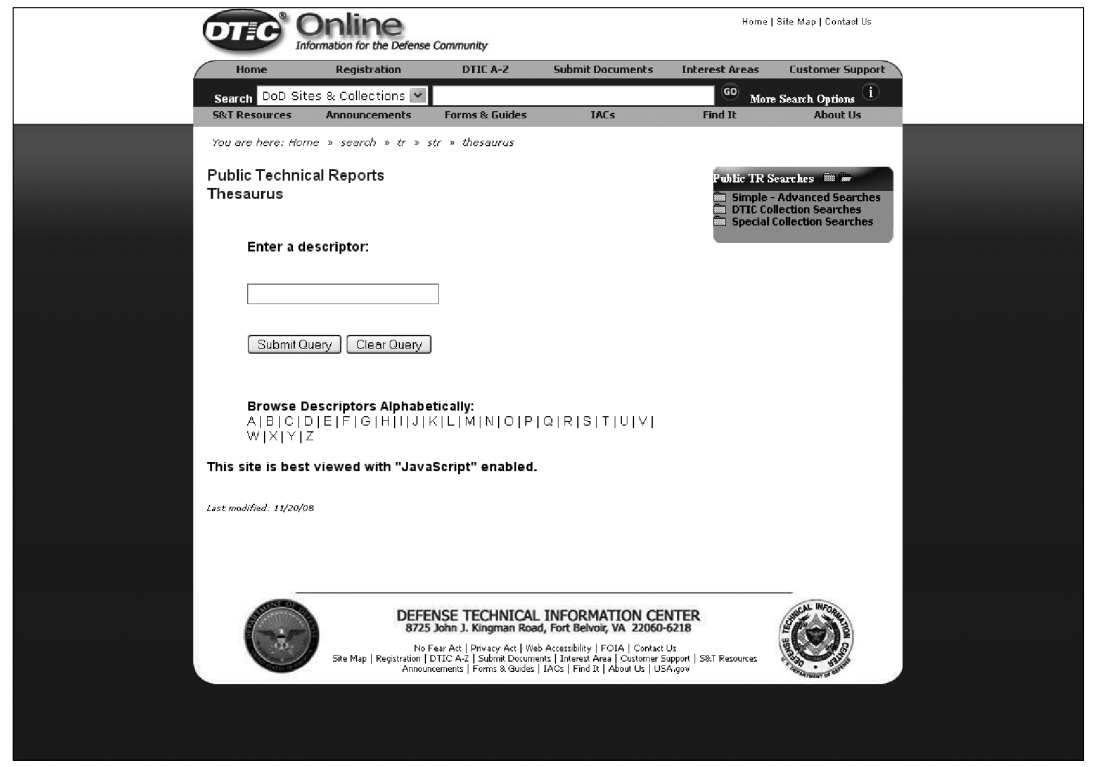




\section{Documentación}

\section{EBSI - Terminologie de base en sciences de l'information bttp://www.ebsi.umontreal.ca/termino/index.htm}

La Escuela de Biblioteconomía y Ciencias de la Información de la Universidad de Montreal incluye en su página web una terminología sobre gestión de la información, archivística y biblioteconomía realizada dentro de un proyecto del comité local de integración pedagógica de dicha universidad entre los años 2000 y 2002. Se trata de una breve terminología (256 descriptores) en francés, publicada para resolver las dudas de los alumnos y profesores de la mencionada universidad en materia de información. La única forma posible de consulta es a través de un índice alfabético en el que no se pueden distinguir tipográficamente los términos admitidos de los no admitidos. La interfaz que presenta la información sobre cada descriptor incluye una definición del término, la fuente de la que se ha extraído, su equivalente en inglés y los términos que conforman sus relaciones semánticas; aunque en bastantes casos no se recoge ningún término en ese apartado.

\section{Infodata-Thesaurus}

bttp://www.infodata-edepot.de/thesaurus/START.HTM

El Centro de Información para las Ciencias y la Praxis de la Información de la Escuela Técnica Superior de Potsdam incluye en su página web el INFODATAThesaurus, un vocabulario controlado sobre ciencias de la información y la tecnología. Este es un tesauro de tamaño medio que contiene 1.756 términos en alemán y que sirve de ayuda en la consulta por materias de la base de datos INFODATA-eDepot, generada por la citada escuela Superior. La consulta del tesauro puede realizarse por medio de un buscador que trunca automáticamente los términos por su parte final, y en un índice alfabético en el que se incluye junto a cada término un enlace a los registros de la base de datos que lo incluyen como descriptor. Los términos se muestran marcados como hiperenlaces para facilitar la consulta.

\section{TCI - Tesauro em Ciência da Informação bttp://www.inf.pucminas.br/ci/tci/index.php?option=com frontpageEItemid=1}

El Tesauro em Ciência da Informação (TCI) se elabora como parte de un trabajo de doctorado sobre creación semiautomática de tesauros realizado por un alumno de la Escola de Ciência da Informação de la Universidade Federal de Minas Gerais. Este vocabulario controlado contiene 2.088 términos (1.891 descriptores distribuidos en quince áreas temáticas y 197 no descriptores) en portu- 
gués sobre biblioteconomía, archivística, documentación, museología y tecnologías de la información. La consulta del TCI puede realizarse en un índice alfabético o en un índice jerárquico, ambos en formato html. La información presentada sobre cada descriptor incluye aquellos términos con los que está relacionado semánticamente y la faceta o grupo jerárquico al que pertenece. La última actualización del TCI se llevó a cabo en mayo de 2006.

\section{TerminosaurusRex - Trex - Die Informationswissenschaft in Begriffen bttp://serverO2.is.uni-sb.de/trex/}

El Terminosaurus Rex es un directorio de recursos electrónicos en línea sobre ciencias de la información que se encuentra alojado en la sede web de la Facultad de Filosofía de la Universidad del Estado de Saarland (Alemania). Los recursos de este directorio se ordenan de acuerdo a la estructura de un microtesauro en alemán sobre ciencias de la información y disciplinas afines creado ad hoc. Este vocabulario controlado contiene 498 descriptores agrupados en tres grandes áreas temáticas. La consulta de su contenido se puede hacer a través de un buscador que trunca de manera automática el principio y el final de los términos empleados en la interrogación; navegando por un índice jerárquico dinámico que se muestra en la parte izquierda de la interfaz; o a través de un índice jerárquico y otro alfabético, ambos estáticos, cuyos enlaces aparecen en la parte superior de la interfaz. La información relativa a cada término se presenta en la parte derecha de la interfaz, e incluye sus relaciones semánticas, una definición del mismo, bibliografía y enlaces a recursos electrónicos relacionados.

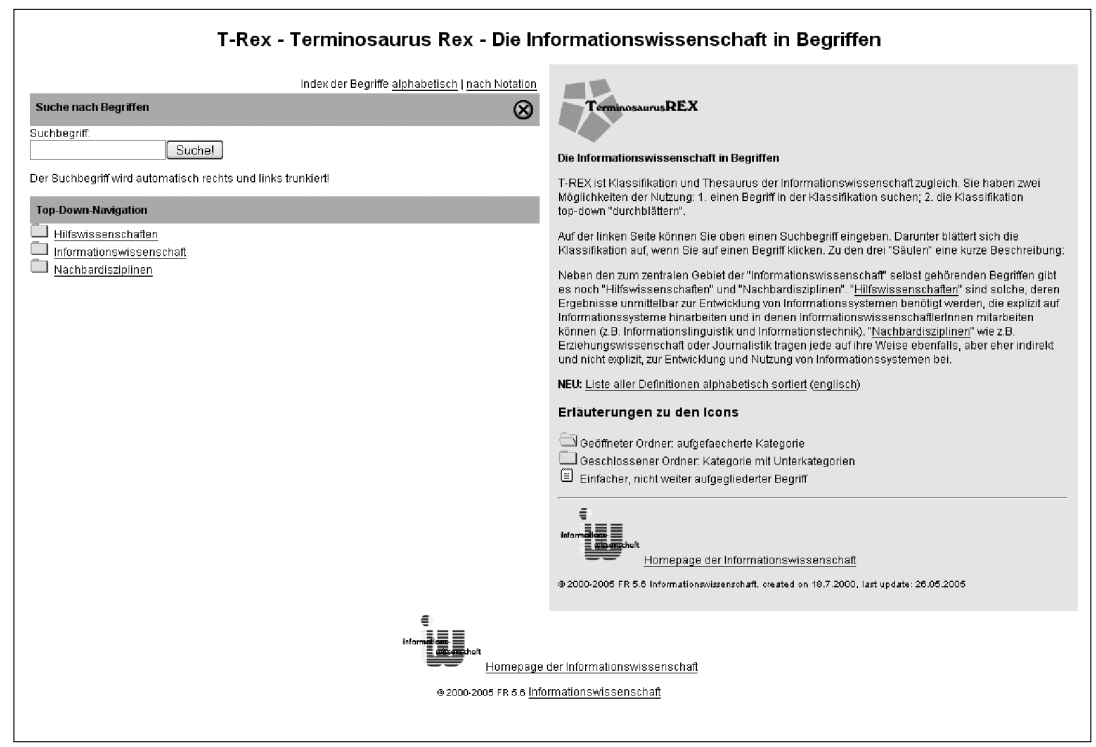




\section{Terms on net (Teron)}

bttp://uexkull.p52s.hio.no/ teron/system.php3?teskode=PEDEspraak=eng

Teron 2 (Terms on the Net) es un proyecto elaborado por alumnos de la Facultad de Periodismo, Bibliotecas y Ciencias de la Información de la Universidad de Oslo para presentar tesauros en la web. Entre los tesauros que alberga dicho proyecto encontramos uno de corta extensión (279 términos) sobre Biblioteconomía y Ciencias de la Información en lengua inglesa. La consulta de su contenido puede hacerse utilizando un buscador que permite el truncamiento anterior y posterior de los términos mediante el símbolo del asterisco (*).

Dentro del proyecto Teron 2 también podemos encontrar un tesauro del Norsk Institutt for Luftforskning sobre investigación del aire en lengua noruega, y el Tesauro Europeo de Educación, elaborado por el Consejo Europeo y la Unión Europea, con una versión integra en noruego y dos versiones reducidas en danés e inglés. El sistema permite realizar búsquedas en todos los tesauros de forma simultánea o en cada uno de ellos. La última actualización de la interfaz del proyecto Teron 2 se realizó en marzo de 1999.

\section{Tesauro de Biblioteconomia y Documentacion bttp://thes.cindoc.csic.es/index BIBLIO esp.html}

Otro de los tesauros editados por el antiguo CINDOC (Centro de Información y Documentación Científica) es el Tesauro de Biblioteconomía y Documentación, cuya autoría se debe a Gonzalo Mochón Bezares y Ángela Sorli Rojo. La finalidad principal de este tesauro es servir de guía en el proceso de indización y recuperación documental de la parte de la base de datos del CINDOC dedicada a la documentación científica. En el tesauro de biblioteconomía y Documentación se recogen 1.483 términos en español (1.099 descriptores distribuidos en doce áreas temáticas, y 384 términos no admitidos) sobre biblioteconomía, documentación, archivística, museología y tecnologías de la información. La consulta de su contenido puede realizarse visitando las áreas temáticas del índice jerárquico; navegando por el índice alfabético, en cual se distinguen tipográficamente los términos admitidos de los no admitidos; o utilizando un buscador al que se accede a través del enlace búsquedas que se encuentra dentro del apartado consultas al tesauro. Esta última herramienta permite la consulta de palabras o fragmentos de palabras, siendo el símbolo \% el carácter de sustitución o truncamiento empleado. La información ofrecida sobre cada término incluye las relaciones semánticas del mismo marcadas como hiperenlaces. La última versión de este Tesauro está fechada en febrero de 2007.

También podemos consultar el contenido de este tesauro en un índice dinámico incluido en el portal de revistas electrónicas españolas sobre información y documentación Temaria (http://temaria.net/tesauro.php), elaborado por la Facultat de Biblioteconomia i Documentació de la Universitat de Barcelona. En este 
caso, el tesauro ha sido utilizado para facilitar el acceso por materias a los artículos incluidos en el Portal.

\section{Tesauro de Ciencias de la Documentación. DOCUTES}

bttp://www3.unileon.es/dp/abd/tesauro/pagina/tesdocumentacion/docutes.htm bttp://eprints.rclis.org/archive/00003067/

El Tesauro de Ciencias de la Documentación (DOCUTES) es un tesauro en lengua castellana dentro de un proyecto de la Universidad de León, dirigido por la Dra. Blanca Rodríguez Bravo. El objetivo principal del tesauro DOCUTES es servir como obra de referencia en las prácticas de indización que realizan los alumnos de la Diplomatura de Biblioteconomía y Documentación de la Universidad de León. Este tesauro se presenta en dos versiones distintas:

Entre los años 2002 y 2003 se elaboró una primera versión del Tesauro DOCUTES que tenía una extensión de 1.478 términos (1.217 descriptores y 261 no descriptores). El contenido de versión se puede consultar en la primera de las dos URLs indicadas más arriba. En la misma se presentan un índice alfabético y un índice jerárquico bajo la denominación de "TERMINOS CABECERA", el cual contiene siete áreas principales. Los términos se presentan marcados como hiperenlaces para mejorar la navegabilidad.

Partiendo de aquella se elaboró, entre los años 2003 y 2005, una segunda versión que contiene 2.196 términos distribuidos en las siguientes categorías: 1.612 son descriptores y 584 son términos no admitidos. El texto completo de esta versión se encuentra disponible en formato pdf en la URL del portal E-LIS que más arriba se indica. La consulta se puede hacer en tres índices diferentes: uno alfabético que presenta las relaciones semánticas de los términos y sus equivalentes en inglés, otro jerárquico con seis familias principales y otro permutado KWOC. También se incluye un índice que recoge los términos en inglés y sus equivalentes en español.

\section{Matemáticas}

\section{BUCM - Tesamat - Biblioteca Complutense bttp://www.ucm.es/BUCM/mat/11187.php}

El Tesamat (Tesauro de Matemáticas) se debe a dos bibliotecarios, que prestan servicio en la sección de proceso técnico de la Biblioteca de Ciencias Matemáticas de la Universidad Complutense de Madrid. Se trata de un tesauro sobre matemáticas en lengua española destinado a servir de elemento de ayuda en la consulta por encabezamientos de materia del Catálogo Cisne de la Universidad Complutense. El Tesamat contiene 1.944 términos (encabezamientos de materia) que se pueden consultar en un índice alfabético simple de términos o en un 
índice alfabético de encabezamientos de materia en el que se incluyen las relaciones semánticas de los encabezamientos y su número correspondiente en los siguientes sistemas de clasificación: CDU, LC y American Mathematical Society (AMS). Este tesauro se actualiza constantemente.

\section{Le Thésaurus de Statistique Canada Statistics Canada Thesaurus bttp://www47.statcan.ca/th r000 f.htm bttp://www47.statcan.ca/th r000 e.htm}

La Agencia Nacional de Estadística de Canadá es un organismo federal que tiene como función principal la producción de estadísticas para ayudar a los habitantes de dicho país a comprender mejor como son su población, sus recursos, su cultura, su economía y su sociedad. Dentro del sitio web de esta agencia canadiense se encuentra el Statistics Canada Thesaurus/Thésaurus de Statistique Canada, desarrollado para facilitar el acceso por materias a los recursos de información sobre estadística elaborados por el mencionado organismo. Este tesauro contiene 5.676 términos, que pueden consultarse en una interfaz en inglés y otra en francés, en cada una de las cuales se incluyen enlaces a un índice alfabético, a un índice jerárquico donde se agrupan los descriptores en 24 categorías temáticas, y un motor de búsqueda que permite la consulta por cadena de caracteres y que añade automáticamente el operador and entre los términos utilizados. La información sobre cada descriptor contiene sus relaciones semánticas, su traducción a la otra lengua en la que el tesauro es operativo y un enlace para consultar la presencia de dicho término en el sitio web, en el catálogo de su biblioteca o en su catálogo de recursos web. La última modificación del contenido se efectuó el 13 de diciembre de 2007.

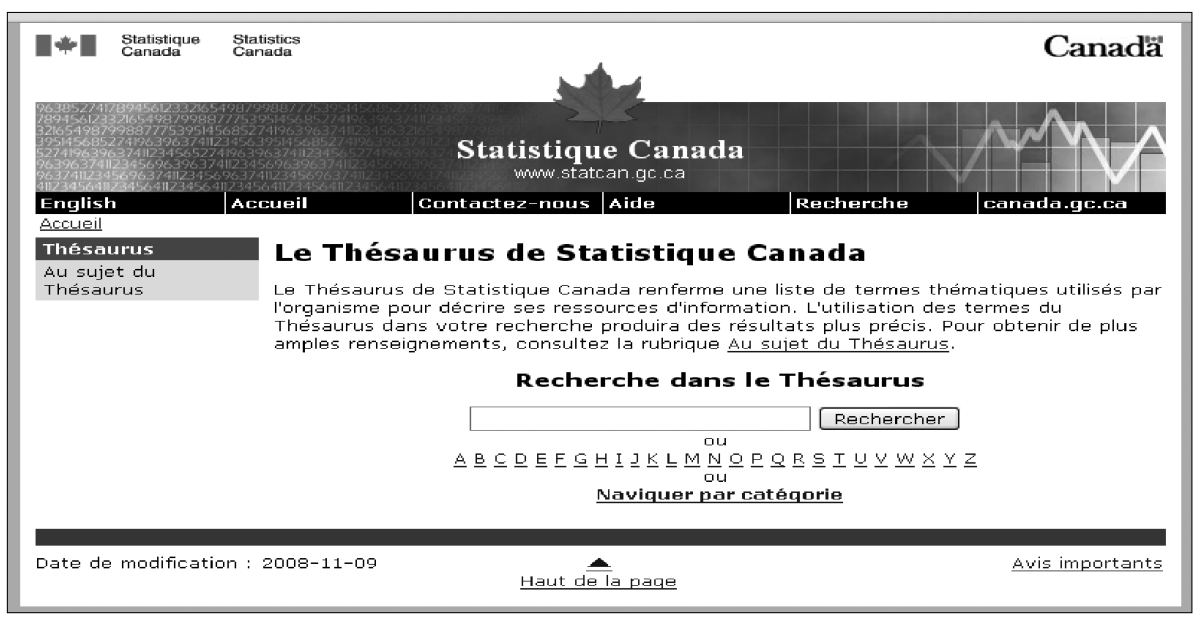




\title{
Tecnologías de la Información y las Comunicaciones
}

\author{
CRTC Thesaurus \\ bttp://www.crtc.gc.ca/thesaurus/10-01E/
}

El sitio web del Consejo de la Radiodifusión y la Comunicación de Canadá (CRTC) alberga el CRTC Thesaurus. Se trata de un tesauro sobre telecomunicaciones y ciencias de la información elaborado para servir como elemento de ayuda en la consulta por palabra clave del contenido del sitio web del CRTC. Dicho tesauro contiene unos 4.000 términos entre descriptores y no descriptores, los cuales se pueden consultar en una versión en francés u otra en inglés, dependiendo de la interfaz de consulta que se escoja. El contenido se presenta en un índice alfabético en el que los términos admitidos y no admitidos se diferencian por la grafía. La información sobre cada descriptor recoge sus relaciones semánticas marcadas como hiperenlaces, su traducción a la otra lengua en la que el tesauro está disponible, la referencia de la fuente de la que ha sido extraído y el área a la que pertenece el término.

\section{Redes de ordenadores \\ bttp://www.um.es/gtiweb/fjimm/tesauro/}

Los miembros del Grupo de tecnologías de la información de la Universidad de Murcia Francisco Javier Martínez Méndez y Juan Carlos García Gómez son los responsables de la creación y mantenimiento del Tesauro de Redes de Ordenadores, que se encuentra alojado en el sitio web de la mencionada universidad. Este vocabulario controlado sobre redes informáticas y protocolos de comunicaciones contiene 262 términos en español, 167 descriptores distribuidos en cinco áreas temáticas y 95 no descriptores. La consulta puede hacerse tres índices en formato html: uno sistemático, otro alfabético y un tercero permutado KWIC. También se puede visualizar el contenido de este tesauro a través de un índice alfabético y otro sistemático, ambos dinámicos, creados con el sistema de gestión de tesauros iSGAT. El Tesauro de Redes de Ordenadores se actualizó por última vez el 20 de febrero de 2006.

\section{Thesaurus Computer Science (alphabetisch) bttp://www.inf.fu-berlin.de/bib/thesaurus-cs.html}

El Computer Thesaurus Science, realizado por Hartwig Baumgaertel como trabajo de diplomatura en la Facultad de Matemáticas e Informática de la Universidad Libre de Berlín, es un tesauro de tamaño medio sobre informática en lengua alemana. Este vocabulario controlado contiene 954 términos, de los cuales 593 son descriptores y 361 son términos no admitidos. El contenido sólo se puede consultar en un índice alfabético en formato html, en el que se especifican 
las relaciones semánticas de los términos. El tesauro se realizó en 1994 y no parece haber sido actualizado desde entonces.

\section{Transportes}

\section{Australian Transport Thesarus bttp://www.arrb.com.au/documents/libraryThesaurus.pdf}

La M. G. Library, entidad dependiente del consorcio australiano ARRB Group, mantiene en su página web el Australian Transport Thesaurus (ATT), un tesauro sobre transporte y carreteras destinado a asistir a bibliotecarios e investigadores en el acceso a la documentación presente en dicha biblioteca. En la confección del mencionado tesauro se han extraídot numerosos términos de indización de la base de datos Australian Transport Index (ATRI). Este tesauro contiene 3.030 términos en inglés, de los cuales 1.836 son descriptores y 1.194 son términos no admitidos como descriptores. La consulta del contenido del tesauro ATT solamente se puede hacer sobre un índice alfabético en formato pdf. La presente edición de este tesauro se realizó en diciembre de 2007.

\section{MDA Railway Object Name Thesaurus bttp://www.mda.org.uk/railway/railcon.htm}

La antigua MDA (Museum Documentation Association), organización británica dedicada a la gestión de colecciones de museos y unidades documentales, incluye en el sitio web de la Collections Trust un tesauro en lengua inglesa sobre construcción y equipamiento de ferrocarriles, elaborado por el Railway Terminology Working Group con el fin de utilizarlo en las labores de indización documental: el MDA Railway Object Name Thesaurus. Este tesauro contiene 4.015 términos, divididos en 3.165 descriptores y 372 no descriptores. La consulta de su contenido puede realizarse en un índice de alfabético en el que se incluyen las relaciones semánticas de todos los términos junto con las fuentes de las que se han extraído y una definición de cada uno de ellos; en un índice simple de términos que contiene el hiperenlace de cada término al índice alfabético; o a través de un índice jerárquico que distribuye a los descriptores en siete áreas temáticas. La presente versión en línea es del año 2002 y nada parece indicar que el contenido de la misma haya sido modificado.

\section{National Transport Library TRT bttp://ntlsearch.bts.gov/tris/trt.do}

La organización estadounidense Transportation Research Borrad ha elaborado una herramienta de lenguaje controlado sobre transporte: el Transportation Re- 
search Thesaurus (TRT). La función de este tesauro es servir de herramienta en la indización y consulta en línea de los registros de la base de datos bibliográfica Transportation Research Information Services (TRIS). El TRT es un macrotesauro en lengua inglesa que contiene 11.823 términos sobre transporte por diferentes medios. La consulta de su contenido puede llevarse a cabo a través de un buscador, que permite el uso de los operadores booleanos and y or, y en el que se muestran los resultados obtenidos en la parte central de la interfaz. El contenido de este macrotesauro también se puede visualizar en un índice alfabético de término o navegando por un índice jerárquico dinámico situado en la parte izquierda de la interfaz. Los descriptores se muestran marcados como hiperenlaces, lo cual permite una consulta más rápida. También se ofrece a los usuarios la posibilidad de descargar el contenido completo en formato XML para su uso fuera de línea. La última actualización de este macrotesauro se realizó en julio de 2007.

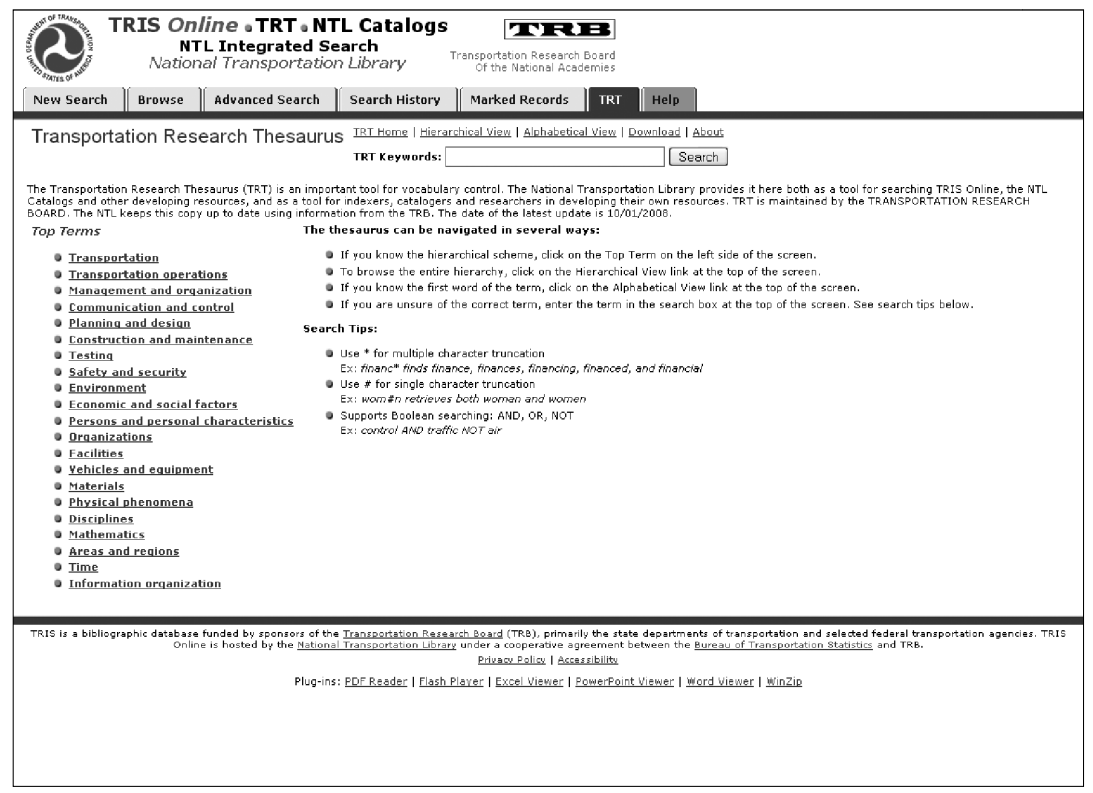

\section{Transport Canada Thesaurus}

bttp://wwwapps.tc.gc.ca/Corp-Serv-Gen/5/Thesaurus/a001-eng.aspx bttp://wwwapps.tc.gc.ca/Corp-Serv-Gen/5/Thesaurus/a001-fra.aspx

El sitio web de la entidad responsable de la gestión de los transportes en Canadá presenta un tesauro basado en el tesauro TRT comentado más arriba. El vocabulario controlado que ahora nos ocupa, el Transport Canada Thesaurus (TCT), ha sido elaborado por la Transport Canada Library para destinarlo a las 
labores de indización y recuperación de los documentos de sus colecciones, aunque también se usa para la localización documental en las páginas web de Transport Canada. El TCT contiene algo menos de 1.900 términos en inglés o en francés, según la interfaz que se elija, sobre vehículos de transporte, ingeniería civil y economía relacionada con el transporte. La consulta del tesauro TCT se hace a través de un buscador en el que se puede interrogar bien por comienzo de término, o bien por cadena de caracteres dentro del término. A la hora de visualizar los resultados se puede seleccionar si se hace como una lista de términos con sus relaciones semánticas o como una lista de términos que incluye los genéricos y los específicos más inmediatos de cada descriptor. La última actualización del contenido de este tesauro fue en marzo de 2008.

UIC

bttp://www.uic.asso.fr/centredoc/spip.php?article27

La Unión Internacional de Vías Férreas (UIC, en francés) dispone de un centro de documentación en línea en el que se incluye un vocabulario controlado: el Transport Thesaurus. Se trata de un tesauro sobre transporte que surgió como resultado de la cooperación entre la Conferencia Europea de Ministros de Transportes y la UIC. El Transport Thesaurus presenta 2.129 descriptores que se pueden consultar en dos índices distintos presentados en formato word: un índice facetado en el que se distribuyen los descriptores en 12 áreas principales, y un índice alfabético que incluye las relaciones semánticas de los descriptores, la indicación de las facetas de los mismos y sus términos equivalentes en francés y alemán. El contenido de este tesauro se publicó en 1995.

\section{Waterways object name thesaurus bttp://www.mda.org.uk/waterw/index.htm}

La Museum Documentation Association (MDA) presenta en su sede web un tesauro sobre transporte fluvial y equipamiento de embarcaciones de transporte. La autoría de este vocabulario controlado recae sobre el MDA Waterways Terminology Working Group, que lo elaboró con el fin de hacer más accesible el contenido de sus colecciones al usuario especializado y también al público en general. La consulta de su contenido puede realizarse bien en un índice de alfabético, el cual incluye las relaciones semánticas de todos los términos, las fuentes de las que se han extraído y una definición de cada término, o bien a través de un índice jerárquico que distribuye los descriptores en cinco áreas temáticas. La presente versión en línea es del año 2002 y nada parece indicar que el contenido de la misma haya sido modificado desde entonces. 
